\title{
Reply to: Venous aneurysms of saphena magna: Is this really a rare disease?
}

\author{
Ch. Tschuor $\cdot$ D. Hahnloser
}

Received: 17 September 2012/ Accepted: 12 October 2012/Published online: 27 November 2012

(C) Springer-Verlag France 2012

\section{Dear Editors,}

We would like to thank our colleagues for their letter to the Editor [1] with regard to the article "A challenging hernia: primary venous aneurysm of the proximal saphenous vein" [2]. We are happy to learn that Donati et al. agreed with us that a primary venous aneurysm of the great saphenous vein is a rare disease and therefore seldom considered in the differential diagnoses of a lump in the inguinal region. The authors of this letter asked several questions and made several comments and vague hypotheses which we would like to reply on and clarify.

- We apologize for not having stated clearly enough that the histological finding confirmed postoperatively the diagnosis of a primary venous aneurysm. We never stated that it is possible to distinguish preoperatively a primary venous aneurysm from a secondary one.

- This young patient had absolutely no clinical signs of varicosis or chronic venous insufficiency (CVI) on either leg. For these reasons, we did not think preoperatively in this direction and did not perform a Doppler ultrasound. Assessing your figure (Fig. 1.1) provided with the patient suffering from a severe CVI, we agree that having this clinical finding and not

This reply refers to the comment available at 10.1007/s10029-012-1003-5.

Ch. Tschuor

Clinic for Visceral and Transplantation Surgery, University

Hospital Zurich, Raemistrasse 100, 8091 Zurich, Switzerland

D. Hahnloser $(\bowtie)$

Clinic for Visceral Surgery, Centre Hospitalier Universitaire

Vaudois, Rue du Bugnon 46, 1011 Lausanne, Switzerland

e-mail: dieter.hahnloser@chuv.ch thinking of a venous aneurysm would be an affront to the ability of a surgeon to draw a clinical diagnosis. The next diagnostic tool in this patient has to be a Doppler ultrasound. However, this was not the picture we had when examining our patient. He showed no signs of CVI.

- By reading the article, the readers notice that the surgical exploration of this lump was done in addition to a planned treatment for perianal condylomata with precancerous cells under general anesthesia in an HIVpositive patient. Surgical exploration was never said to be the diagnostic tool of choice for venous aneurysms.

- In their letter, the authors raised the point: “...looking at the published image of the upper thigh, it is very clear that this 'hernia' is surely not an inguinal hernia but could be, in the best hypothesis, a femoral one...". Of course, we agree that the location and clinical presentation of this lump is rather atypical for any kind of hernia. Therefore, we stated in the article that this finding can be "confused" with a hernia and pointed out "rather with a femoral hernia". Besides this, we would like to emphasize on the fact that there have been several publications discussing the problem of confusing a venous aneurysm with an inguinal or femoral hernia $[3,4]$.

- Our short article had no intention to describe a rare disease, nor the correct workup or all treatment options. However, this article is a case report. The intention of this case report was to share a surprising and incidental intraoperative finding of an unusual clinical presentation of a seldom and rarely in the differential diagnoses considered disease. According to our understanding, a case report should report the true course of a case even though it was not running optimally. In our wide grades, it is common to learn from morbidity and 
mortality conferences where such cases are discussed, so everyone can learn something. In retrospective, one always knows more!

In the spirit of a cooperative reply, we would like to thank the editors for the possibility to answer for the raised points and commenting on the vague hypotheses.

Cordiali saluti dalla Svizzera,

Christoph Tschuor

Dieter Hahnloser

Corrigendum (1): On the first page, case report, section 2: However, since surgical treatment for condylomata accuminata under general anesthesia had not yet been planned...

\section{Conflict of interest None.}

\section{References}

1. Donati M, Biondi A, Brancato G, Donati A, Basile F (2012) Venous aneurysms of saphena magna: is this really a rare disease? Hernia. doi:10.1007/s10029-012-1003-5

2. Tschuor C, Dindo D, Clavien P, Hahnloser D (2012) A challenging hernia: primary venous aneurysm of the proximal saphenous vein. Hernia. E-pub 17 Mar

3. Majeski J (2002) Surgical repair of primary saphenous vein aneurysm of the proximal leg after initial presentation as an inguinal hernia. Am Surg 68:999-1002

4. Marcucci G, Accrocca F, Antignani PL, Siani A (2010) An isolated aneurysm of the thigh anterolateral branch of the greater saphenous vein in a young patient presenting as an inguinal hernia. Interact Cardiovasc Thorac Surg 10:654-655 